\title{
Dynamic response mechanism of thin-walled metallic defensive structure from explosion in transportation
}

Shujian Yao ${ }^{a, b, c, d}$, Nan Zhao ${ }^{a}$, Duo Zhang ${ }^{e}$, Zhonggang Wang $^{e}$ and Zhen Chen ${ }^{a, b, *}$

a. School of Traffic \& Transportation engineering, Central South University,

Changsha, China

b. Key Laboratory of Traffic Safety on Track, Ministry of Education; Changsha, China

c. Joint International Research Laboratory of Key Technology for Rail Traffic

Safety, Changsha, China

d. National \& Local Joint Engineering Research Center of Safety Technology for

Rail Vehicle, Changsha, China

e. College of Science, National University of Defense Technology, Changsha, China.

*Correspondence: chenzhen88@csu.esu.cn

\begin{abstract}
Defensive structure is important in transportation field for kinds of intentional or unintentional explosion. Structures subjected to unconfined and confined explosion will bear different blast loads and their dynamic responses are different. The present work focus on the dynamic response mechanism of steel plate under unconfined and confined blast loads through both numerical and experimental studies. In the experiment, Digital Image Correlation (DIC) technique was applied to record and analyze the dynamic response process of large-scale field blast test. The DIC measured curve and the numerical calculated curves agrees well in both the trends and the peak values. Then, the dynamic response mechanism of steel plate under unconfined blast (UB) load and confined blast (CB) load were compared and discussed. Results show that the dynamic response of plate can be divided into three phases under both UB and CB loads, while with different mechanism. In phase I, plastic hinge starts from the center and moves to the boundary in UB condition, while in case of CB, plastic hinge occurs close to the boundary and moves in the opposite direction. In phase II, two plastic hinge lines propagates towards each other, and a platform exists between the boundary and the central area remains undeformed in UB condition, while in $\mathrm{CB}$ condition, larger deformation in peripheral region rather than central area produces.
\end{abstract}


Keywords: Unconfined blast; Confined blast; Digital Image Correlation; Dynamic response; Blast loading.

\section{Introduction}

Safety is the eternal theme of transportation. In recent years, frequently serious disasters related to explosion happened around the world in kinds of transportation equipment such as buses, trains and infrastructures. Some are caused by deflagration content unintentionally carried by passengers; some even caused by terrorist activities. To shield such attacks, defense technology or infrastructures (see Fig.1), particularly with light-weight efficient defensive structures should be developed. In addition, some particular reinforcement design should also be considered for ship cabins and railway vehicles.

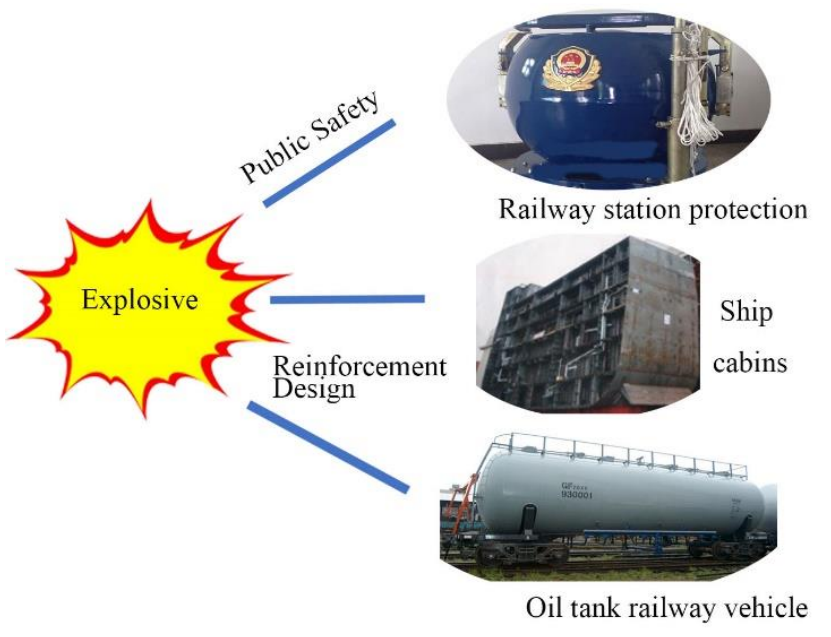

Fig.1 Defensive technique applicated in transportation engineering field.

For this purpose, the vulnerability of the structures needs to be adequately investigated firstly. As the existing finding's shown, structures subjected to unconfined and confined explosion bears different blast loads and their dynamic responses are different [1]. Under unconfined blast, the structure performs different dynamic response which hugely depends on the explosive source distance (i.e., close-in airblast [2], and faraway airblast), shield materials (i.e. thin aluminium, steel plates [3]) as well as the structure patterns. More studies were focused on the failure of the shield plates and effect of stand-off distance, as done by Nurick et al. [4-5], Bonorchis et al. [6-7], Chung et al. [8-9], Jacob et al. [10], respectively. As found that in these studies, the whole process can be categorized into three distinct phases, including, a) Phase " $i$ " , the 
expansion of explosion, from time of detonation to its interaction with the structure; $b$ ) Phase "ii", explosive plate interaction; and c) Phase "iii", expansion of the explosion from time of separation from plate to expansion of the plate equilibrium [11]. In addition, to further explain the dynamic mechanical behavriours, further effectively predict response modes, some theoretical analyses were also developed [13], analytical solution were obtained in good agreement with numerical and experimental observation. These constructive methodologies were also extended to analyze underwater shock wave loading [14]. As aforementioned, the flexural waves emanate from the plate boundary and propagates towards the plate center [15]. Thanks for the development of innovative material technologies, kinds of composited light-weight structures were also investigated [16-20] under impact loads.

Unlike the previous unconfined blast situation, a Confined blast (CB) means that explosion occurs within a structure, which limits the propagation of blast wave. Existing researches have shown that $\mathrm{CB}$ waves are more complicated and more destructive than that of unconfined blast (UB) with equivalent explosive charge [2123]. Whilst the dynamic response mechanism of structures under CB loads and the differences between CB and UB loads are less investigated.

In order to investigate the dynamic response mechanism, effectively measuring the dynamic response process is necessary. However, traditional measurement methods are difficult in measuring the 3D dynamic response and obtain full-field data of blast loaded plate [24-26]. Fortunately, an advanced method using Digital Image Correlation (DIC) technique [27-28] is adept in measuring the 3D dynamic response of structures under impact loading, and it has been turned out to be a reliable tool for full-field transient plate deformation measurements during blast loading, with high accuracy and efficiency [29]. Rigby et al. [30] studied the transient deformation of plates subjected to near-field explosive blasts by using DIC technique, the flexural waves were observed. Spranghers et al. [31] and Kumar et al. [32] employed the DIC technique in the study of the dynamic response on aluminum panels. While, the application of the 3D-DIC technique in the large-scale field test of confined blast is inadequate.

The present work aims at investigating on the dynamic response mechanisms of steel plate under both CB and UB loads, field blast test is conducted and 3D-DIC technique is employed together with elaborate numerical simulations, which paves a way on crashworthiness designing of the kinds of defensive structures. 


\section{Experiment in confined case}

\subsection{Setup and DIC technique}

To create a confined blast loading condition, a steel box chamber was designed and manufactured, as shown in Fig.2, of the (a) schematic experiment set up of the chamber and (b) TNT, respectively. The chamber is made up of Q235 mild steel with yield strength of $370 \mathrm{MPa}$ and Young's modulus of $208 \mathrm{GPa}$, respectively, which are obtained through tensile test with the material test system.

The side length ( $L$, see Fig.2(a)) of $600 \mathrm{~mm}$ are considered in the test. One fifth extra length $(120 \mathrm{~mm}, L / 5)$ of the box side length are welded to every side plate as boundary plates for the purpose of constraint. The plate thickness is $4 \mathrm{~mm}$ and the final dimension of the chamber is $840 \mathrm{~mm} \times 840 \mathrm{~mm} \times 840 \mathrm{~mm}$ as shown in Fig. 2. In the present study, TNT is casted into a cube with a density of $1.5 \mathrm{~g} / \mathrm{cm}^{3}$ and weight $98.4 \mathrm{~g}$. And the TNT explosive is suspended in the inside center of the chamber (see Fig.2).
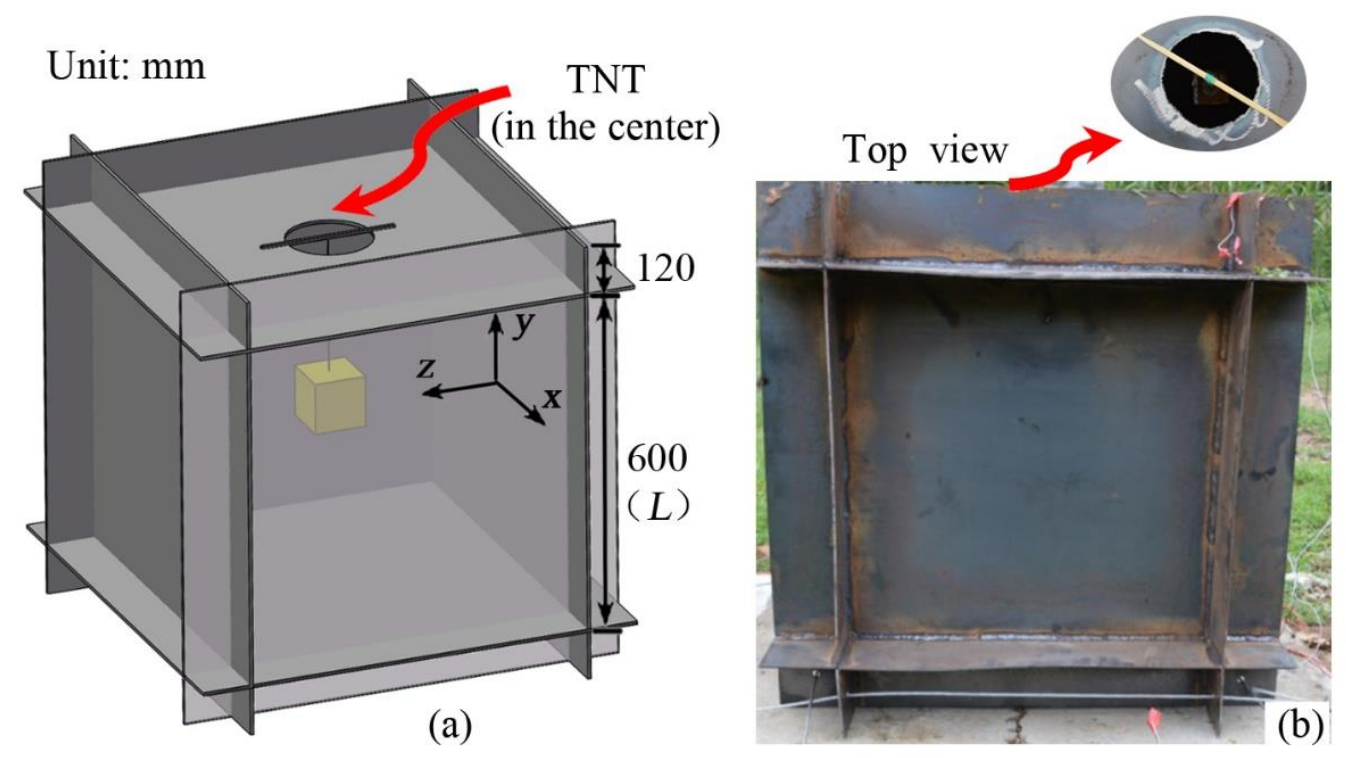

Fig. 2 (a) schematic experiment set up of the chamber and (b) TNT.

DIC technique can record the full-field and 3-D surface deformation with high spatial resolutions and excellent accuracy [29-30]. In this experiment, 3D-DIC technique is applied to record the dynamic response process of the target steel plate. Before the test, two high-speed digital cameras are placed in the front of the target plate arranged at a specific angle to record synchronized images (see Fig. 3). 


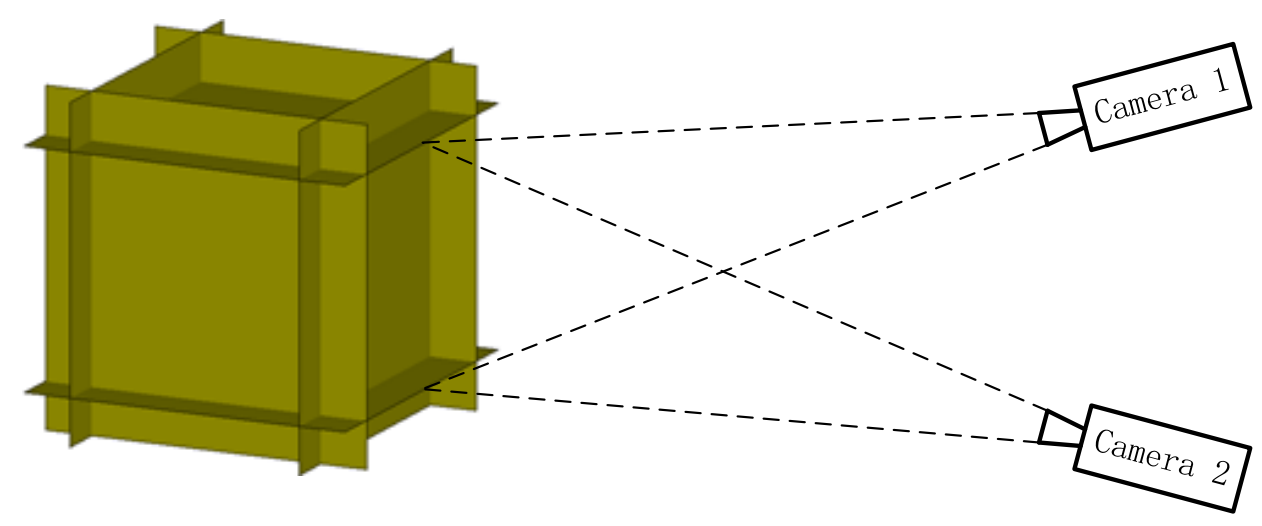

Fig. 3 The layout of high-speed digital cameras.

The DIC workflow is depicted as Fig. 4. The outside surface of the target plate is painted with white background and stochastic black speckle patterns to obtain high contrast images, the area ratio of black to white is about half and half. At the same time, calibration panel with white background and black spots is designed and manufactured for the purpose of obtaining calibration images, the panel used in the calibration process consists of a series of circular dots arranged in a specific form. Then, two high-speed digital cameras (Photron FASTCAM SA5, see Fig.3) were used in a stereo configuration to record synchronized images. These two high-speed cameras were placed in suitable positions with reasonable distance and view angle. And then, synchronized calibration images were recorded. During the calibration process, the panel is moved disorderly around the setup position of the target side plate and about 20 synchronized calibration images were acquired by both cameras. After that, the field blast test was conducted and the dynamic response images of the target plate are recorded synchronously. The post data analysis processing is performed with the standard image correlation software VIC-3D. It matches common pixel subsets of the random speckle pattern between the deformed and un-deformed images. The matching of pixel sets was used to calculate the three-dimensional location of the target plate throughout time. Finally, the full-field shape and deformation are obtained by mapping all of the speckle patterns. 


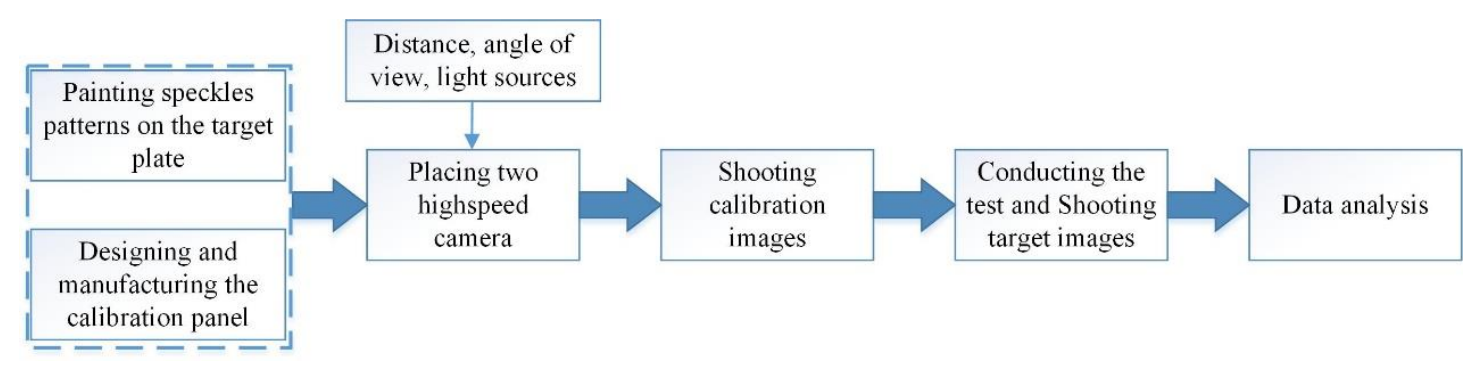

Fig. 4 Diagram of DIC workflow.

\subsection{Experimental results}

The dynamic deformation processes of fully confined blast obtained through DIC technique are shown in Fig. 5, in which the value is a absolute result considering the bending displacement (the orginal value is negative according to location in Fig.2). With the detonation of the TNT explosive, deformation occurs within a very short time at the central area. The plate center deflection increases to $7 \mathrm{~mm}$ at $t=0.52 \mathrm{~ms}$ (see Fig. 5(a)). Then the shock wave propagates to the box corner, and the corner area of side plate deformed (as shown in Fig. 5(b)). As time goes on, the deformation further developed, the corner deformation mode was replaced by center deformation mode (see Fig. 5(c)). The maximum displacement appears at $t=2.27 \mathrm{~ms}$, up to $48.30 \mathrm{~mm}$ as shown in Fig. 5(d).

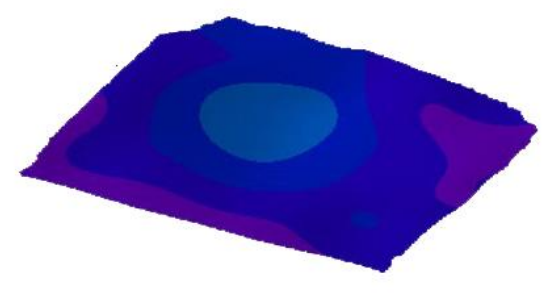

(a) $\mathrm{t}=0.52 \mathrm{~ms}$

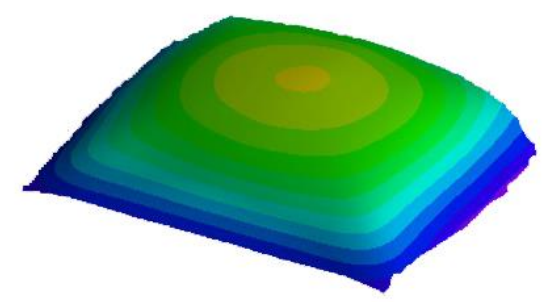

(c) $\mathrm{t}=1.47 \mathrm{~ms}$

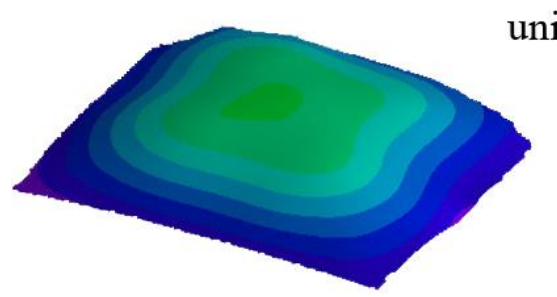

(b) $\mathrm{t}=1.17 \mathrm{~ms}$

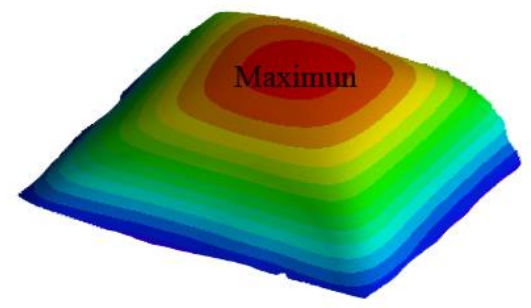

(d) $\mathrm{t}=2.27 \mathrm{~ms}$ unit:mm

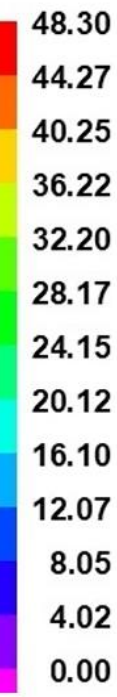

Fig. 5 DIC results of dynamic response process of plate CB (value should be negative according to location in Fig.2). 


\section{Numerical simulation}

\subsection{Finite element model}

Finite element (FE) models of steel plate under UB and CB load were built by using ANSYS software ${ }^{\circledR}$. The model for UB load is showed in Fig. 6(a), in which the plate is fixed in both directions with a thickness of $4 \mathrm{~mm}$, and the explosive is placed above the plate center with a distance of $300 \mathrm{~mm}$. The model for CB load is showed in Fig. 6(b). A steel box model with a clear side length of $600 \mathrm{~mm}$ (as shown in the right picture of Fig. 6(b)) is built to simulate a fully confined blast condition. The explosive is placed in the center of the box model.

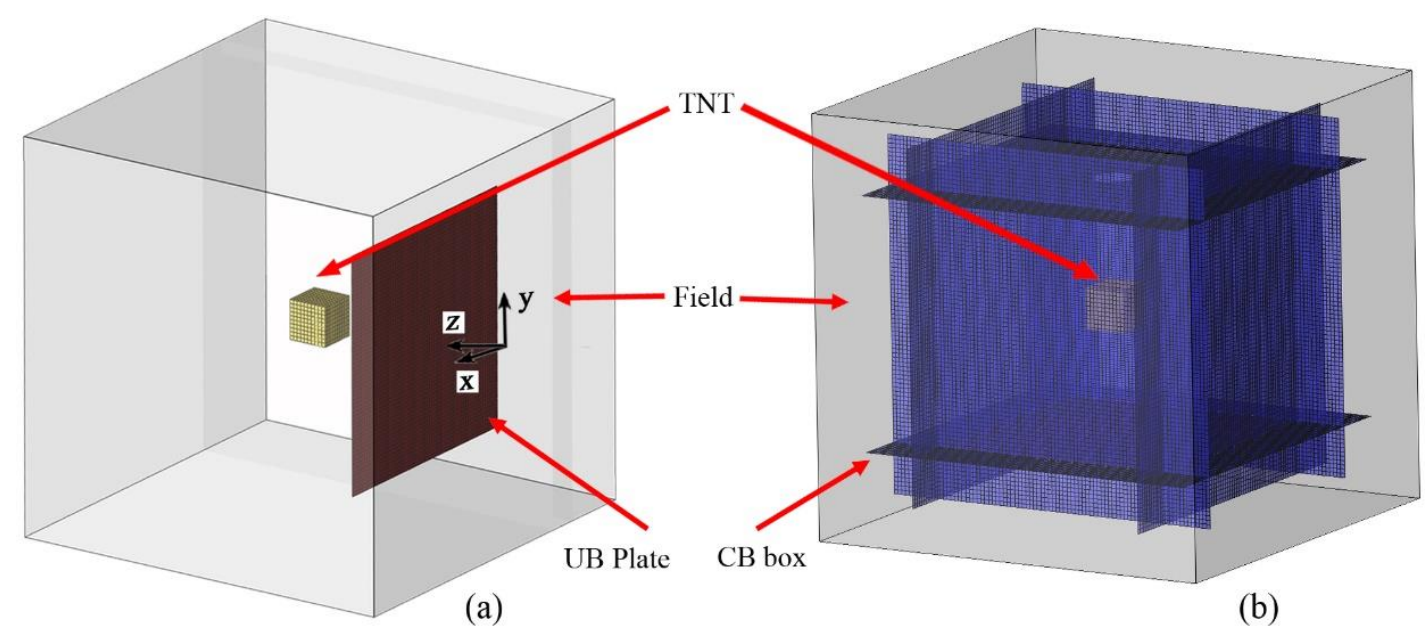

Fig. 6 Finite element models of (a) UB and (b) CB.

Both in the UB model and the CB model, the explosives are defined in the region of air by using the keyword *INITIAL_VOLUME_FRACTION_GEOMETRY. The coupling of blast wave and structure is considered through adopting the fluid-structure interaction (FSI) algorithm. Artificial bulk viscosity $q$ is applied to avoid numerical oscillations in the practically discontinuous rise of blast waves, and $q$ can be calculated as

$$
\begin{cases}q=\rho l\left(c_{0} l\left|\dot{\varepsilon}_{k k}\right|^{2}-c_{1} \alpha\left|\dot{\varepsilon}_{k k}\right|\right) & \dot{\varepsilon}_{k k}<0 \\ q=0 & \dot{\varepsilon}_{k k}>0\end{cases}
$$

where $c_{0}$ and $c_{1}$ are dimensionless constant, they meet $c_{0}=1.50, c_{1}=0.06 . \rho$ is present density, and $l=\sqrt[3]{V}$ ( $V$ is the volume) is the characteristic length, $\alpha$ is the localized sound velocity, $\dot{\varepsilon}_{k k}$ is the tensor of strain rate. With the artificial bulk viscosity $q$, the calculation of tress $\sigma_{i j}$ is as follows 


$$
\sigma_{i j}=S_{i j}+(P+q) \delta_{i j}
$$

where $P$ is the pressure and $S_{i j}$ is deviant stress tensor.

In the FE model, the explosive and air are meshed with the eight-node Eulerian element SOLID164, while the structures are meshed with four-node element SHELL163. The Arbitrary-Lagrange-Euler (ALE) algorithm is applied to model the air and TNT explosive, while Lagrange algorithm is used for the steel plate and box model. Since the calculation accuracy of the shock wave state parameters are highly dependent on the mesh size of the Eulerian element, analysis on the mesh size sensitivity were conducted as shown in Fig. 7. As can be seen in Fig. 7, the calculated results converge gradually at $6 \mathrm{~mm}$ mesh size to $4 \mathrm{~mm}$ in both blast wave pressure (see Fig. 7 (a)) and structural response (see Fig. 7 (b)). Hence, the mesh size of Eulerian element is designed as $4 \mathrm{~mm}$, and the mesh size for structure is $5 \mathrm{~mm}$. The total element number in CB model for shell and solid are 169740 and 12167000, respectively. The air model for UB load is the same with CB load and the element number for plate in the UB model is 28224 .
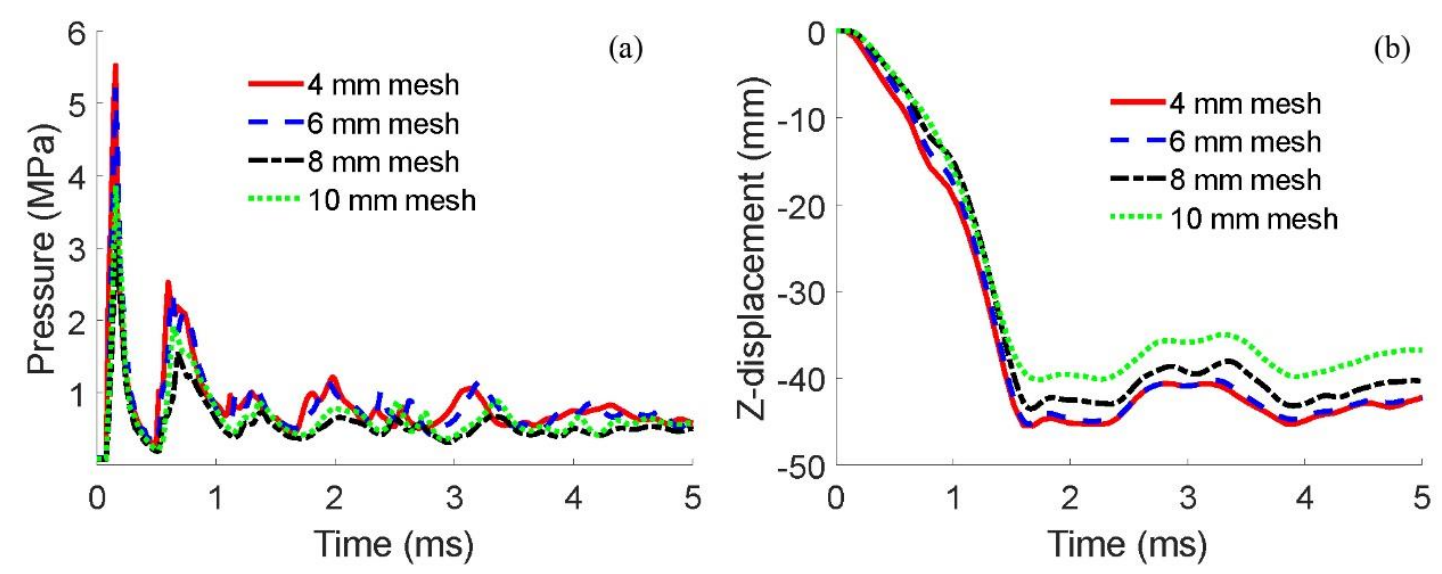

Fig. 7 Mesh size sensitivity analysis: (a) pressure at the inner face of plate center; (b) result displacement of the plate center.

\subsection{Material and propeties}

Numerical method, using finite element method (FEM) programs, has become a common tool in the investigation of structural impact [7, 26]. LS-DYNA has been widely applied for its ability to solve problems with large plastic deformation and can consider the strain rate effect [33]. In the numerical models, air is assumed to ideal gas that modeled by linear-polynomial EOS and linear in internal energy [34-35]. The 
explosive is viewed as a high explosive burning material and the Jones-Wilkins-Lee (JWL) equation of state (EOS) is selected to simulate the pressure of the explosive explosion. It can be expressed as follows:

$$
P=A\left(1-\frac{\omega}{R_{1} V}\right) e^{-R_{1} V}+B\left(1-\frac{\omega}{R_{2} V}\right) e^{-R_{2} V}+\frac{\omega E}{V}
$$

where $A, B$ are linear blast parameters; $\omega, R_{1}$ and $R_{2}$ are nonlinear parameters; $V$ is relative volume and $E$ is specific internal energy. TNT is selected for explosive charge in the current tests, the parameters of material model and EOS can be referred in Refs. [34-35].

The steel material is modeled by Johnson and Cook (J-C) model [35] which has been shown of accurate predictions of steel structures subjected to blast loads. The general equation of J-C model is presented as Eq. (4). The parameters for steel are listed in Table $1[9,34-36]$.

$$
\sigma_{y}=\left(A+B \bar{\varepsilon}^{p^{n}}\right)\left(1+C \ln \varepsilon^{*}\right)\left(1-T *^{m}\right)
$$

The Gruneisen equation of state for steel as compressed materials is presented as follows:

$$
p=\frac{\rho_{0} c^{2} \mu\left[1+\left(1-\frac{\gamma_{0}}{2}\right) \mu-\frac{a}{2} \mu^{2}\right]}{\left[1-\left(s_{1}-1\right) \mu-s_{2} \frac{\mu^{2}}{\mu+1}-s_{3} \frac{\mu^{3}}{\mu+1}\right]^{2}}+\left(\gamma_{0}+a \mu\right) e_{t}
$$

and for expanded materials as:

$$
p=\rho_{0} c^{2} \mu+\left(\gamma_{0}+a \mu\right) e_{t}
$$

where $s_{1}, s_{2}$, and $s_{3}$ are the coefficients of the slope of the $v_{s}-v_{p}$ curve; $\gamma_{0}$ is the Gruneisen gamma; $a$ is the first order volume correction to $\gamma_{0} ; \mu=\rho / \rho_{0}-$ 1.

Table 1. Parameters of J-C material model and Gruneisen state equation for steel.

\begin{tabular}{cccccccccc}
\hline $\mathrm{J}-\mathrm{C}$ & $\rho\left(\mathrm{kg} / \mathrm{m}^{3}\right)$ & $E(\mathrm{GPa})$ & $G(\mathrm{GPa})$ & $v$ & $A(\mathrm{MPa})$ & $B(\mathrm{MPa})$ & $n$ & $c$ & $m$ \\
model & 7800 & 210 & 80.8 & 0.3 & 370 & 438 & 0.60 & 0.01 & 0.669 \\
\hline Gruneisen & $C(\mathrm{~m} / \mathrm{s})$ & $\mathrm{S} 1$ & $\mathrm{~S} 2$ & $\mathrm{~S} 3$ & $\gamma_{0}$ & $a$ & & & \\
EOS & 4569 & 1.49 & 0 & 0 & 2.17 & 0.46 & & & \\
\hline
\end{tabular}

\subsection{Experimental validation}


The deformation features of steel plate under CB load obtained from both experimental and numerical results are shown in Fig. 8. It can be founded that outward bulging in the central area of side plates and in-plane buckling in the middle position of boundary plates in both blasts tested and simulated results. The comparison results indicate that the numerical model simulates the deformation features well in the issues of steel plates subjected to blast loads.
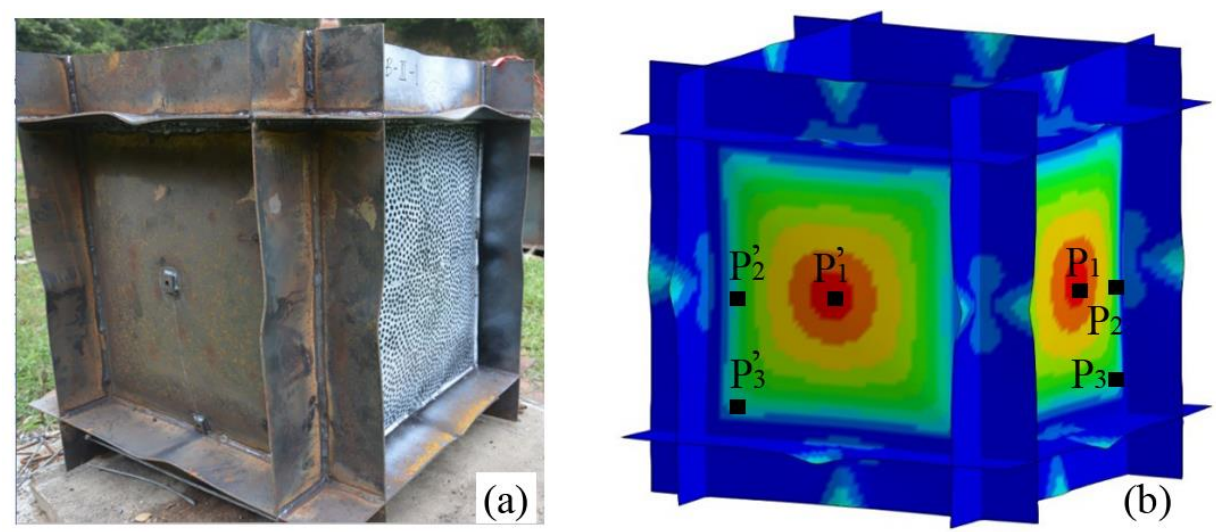

Fig. 8 Deformation features of experimental and numerical result: (a) experimental result; (b) simulated result.

Through the post-processing of deformed and un-deformed image pairs obtained from the two high speed cameras, the deformation value on the considered side plate in every time step can be calculated through DIC technique. Hence, the deflection-time curves of the studied side plate can be obtained. Fig. 9 shows the comparison of deflection-time curve in the side plate center between DIC measured (the red curve) and numerical calculated (the blue curve). The DIC measured curve and the numerical calculated curves are similar in both the shape and the extreme value. In addition, the period of oscillation of numerical result fits well with the DIC result. 


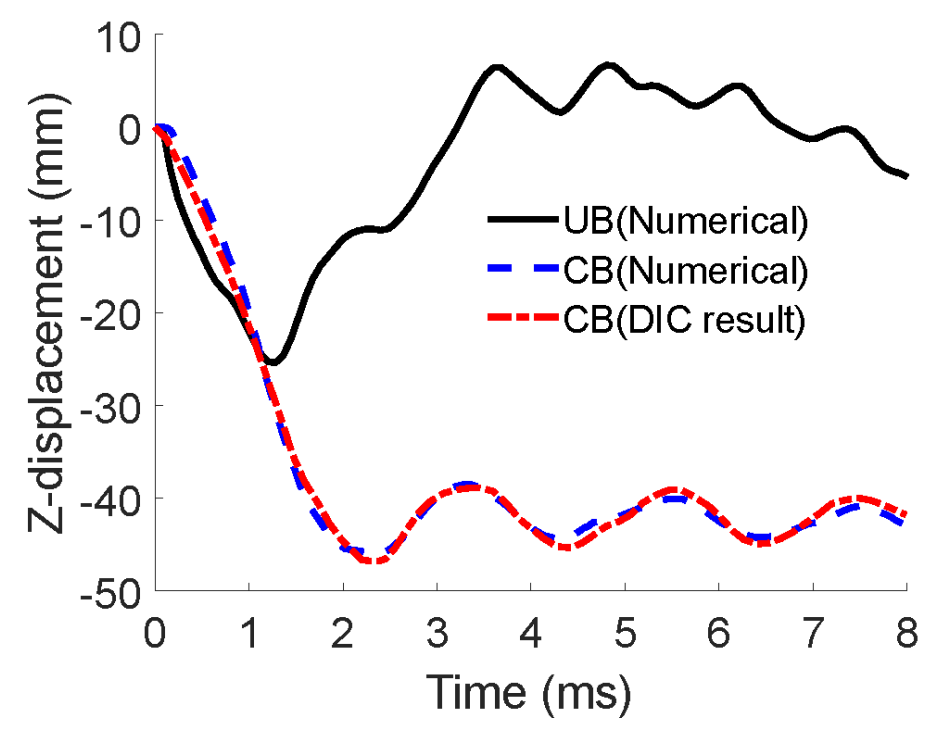

Fig. 9 Comparison of plate center deflection-time curves.

Based on the 3D displacement data of the target plate is obtained, the strain-time data can be calculated by least squares fitting of a quadratic function to each component through the post process of DIC technique. Fig. 10 shows the comparison of strain-time curves of typical positions between DIC measured (the solid line curves) and FEM calculated (the dash line curves). In which the $\mathrm{P}_{1}$ lies at the center of the target plate, $\mathrm{P}_{2}$ near the side length and $\mathrm{P}_{3}$ lies at one of the diagonals of the rectangular side plate, the detailed positions of $\mathrm{P}_{1}-\mathrm{P}_{3}$ are introduced in Fig. 8(b). Additionally, for convenience of finding the points of $\mathrm{P}_{1}$ ', $\mathrm{P}_{2}$ and $\mathrm{P}_{3}$, in Fig.8(b), $\mathrm{P}_{1}$ ', $\mathrm{P}_{2}$ ' and $\mathrm{P}_{3}$ ' also marked to shown the relative position with each other. The strain results show that the curves of FEM results agree well with the DIC results. The approximate strain at $z$ direction (see Fig.2) for plate center is about 0.0045 (see $\mathrm{P}_{1}$ curves in Fig. 10). The strain of $\mathrm{P}_{3}$ is much larger than that of $\mathrm{P}_{1}$ and $\mathrm{P}_{2}$. In conclusion, the numerical model built in the present study is capable of simulating the dynamic response of plate under blast loads well and shows adequate accuracy. 


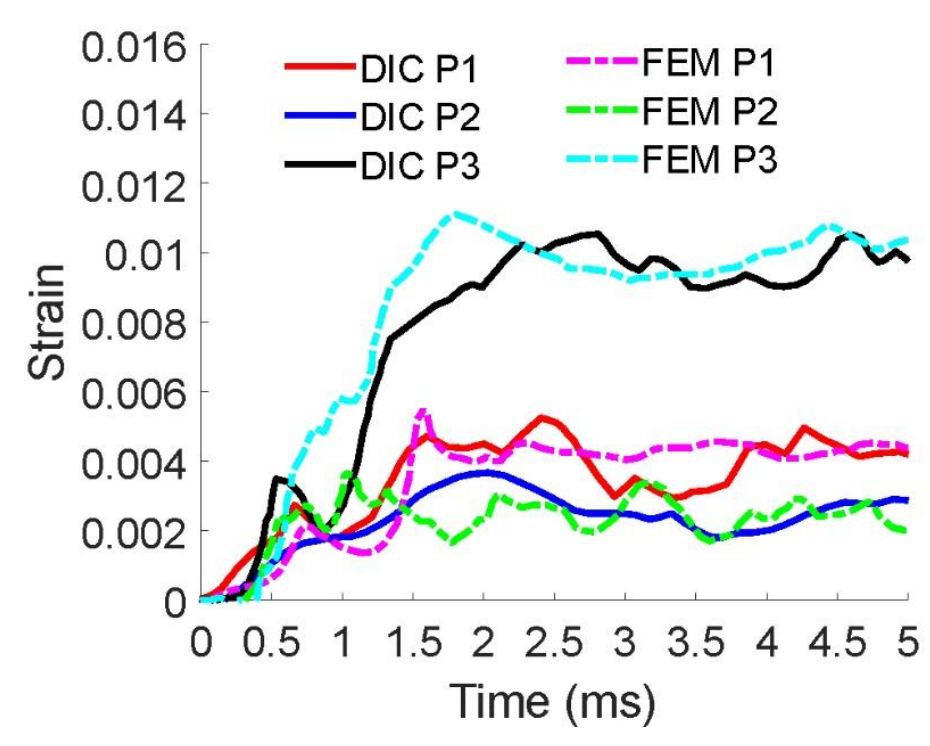

Fig.10 Comparison of $z$-direction strain in typical positions.

\subsection{Results and correlation}

The dynamic response of steel plate under UB load and CB load (the models were shown in Fig. 6) were calculated based on the previous introduced numerical method. The pressure-time curves of UB model and CB model at the target plate center were given in the Fig. 11. There was only one impulse load in UB model with a peak value of 5.4 MPa. While, in the CB model, several smaller peaks followed the first impulse were observed due to the reflection of shock waves in the confined model. The plate center deflection-time curves of the two finite element models were campared in Fig. 9. The maximun deflection of UB model (the black curve) was much smaller than that of $\mathrm{CB}$ model (the blue and red curves), and springback response occurring after maximum deflection in the UB loading. The springback phenominon is called "counterintuitive behaviour" which are studied by several scholars [37-39], their works show that the plates or shells can even reach a final deflection in a direction opposite to the direction of the pulsive loads in specific loading conditions. The results in Fig. 9 and Fig. 11 showed that the dynamic response of steel plate under UB load was very different from the CB load. 


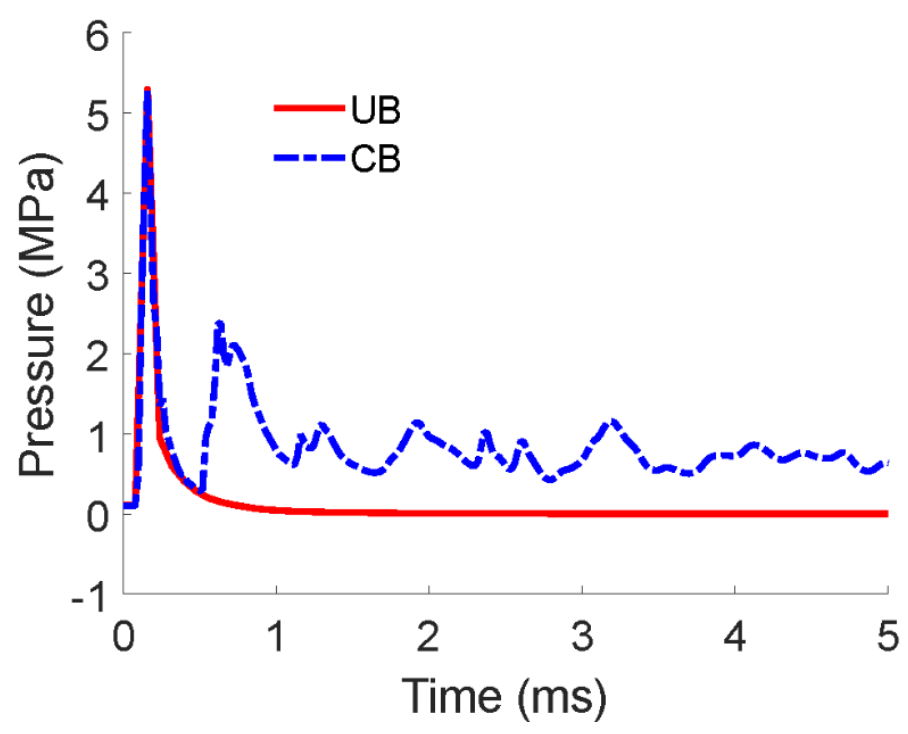

Fig. 11 Pressure-time curves of UB and CB model at the target plate center.

Fig. 12 and Fig. 13 showed the dynamic response process of plate under unconfined and confined blast load, respectively. And the cross-section deformation profiles of UB model at different typical times were depicted in Fig. 14. As shown in Fig. 12, when subjected to unconfined blast load, the central area of the plate deformed firstly, and the response spreads along the radius direction with the central deformation increased (as shown in the $t=0.24 \mathrm{~ms}$ of Fig. 12). And then, plastic deformations were observed both in the central area and around the boundaries as shown in the $t=$ $0.40 \mathrm{~ms}$ of Fig. 12). While the district between the central and the boundaries remain undeformed (see the platform in the cross-section deformation profiles of Fig. 14). After that, the plastic deformation along the boundaries developed towards to the center while the central deformation towards to the boundaries (as shown in Fig. 14, the length of the platform gets shorter from $t=0.24 \mathrm{~ms}$ to $t=0.40 \mathrm{~ms}$ ). At $t=0.64 \mathrm{~ms}$, the plastic deformation spread to all undeformed region (the platform disappeared in Fig. 14). As time goes by, the deformation developed in the central area, the maximum deflection reached $25 \mathrm{~mm}$ at $t=1.20 \mathrm{~ms}$. 


$t=0.12 \mathrm{~ms}$
$\underline{t=0.24 \mathrm{~ms}}$
$t=0.40 \mathrm{~ms}$
$t=0.64 \mathrm{~ms}$
$t=0.88 \mathrm{~ms}$
$t=1.20 \mathrm{~ms}$

unit: $m$

2.540e-02

$2.286 \mathrm{e}-02$

2.032e-02

$1.778 \mathrm{e}-02$

$1.524 \mathrm{e}-02$

$1.270 \mathrm{e}-02$

$1.016 \mathrm{e}-02$

7.621e-03

$5.081 \mathrm{e}-03$

$2.540 \mathrm{e}-03$

$0.000 \mathrm{e}+00$

Fig. 12. Resultant displacement of middle sectional under UB.

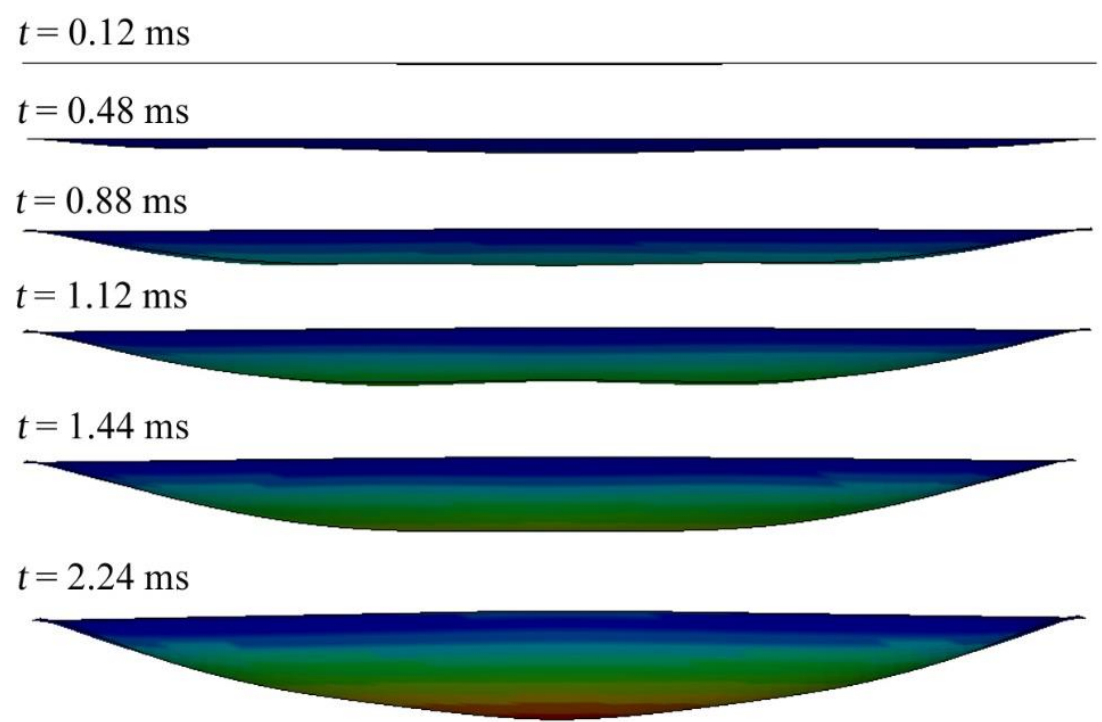

unit: $m$

4.752e-02

$4.277 \mathrm{e}-02$

$3.802 \mathrm{e}-02$

$3.326 \mathrm{e}-02$

2.851e-02

2.376e-02

1.901e-02

$1.426 \mathrm{e}-02$

9.504e- 03

$4.752 \mathrm{e}-03$

$0.000 \mathrm{e}+00$

Fig. 13. Resultant displacement of middle sectional under CB. 


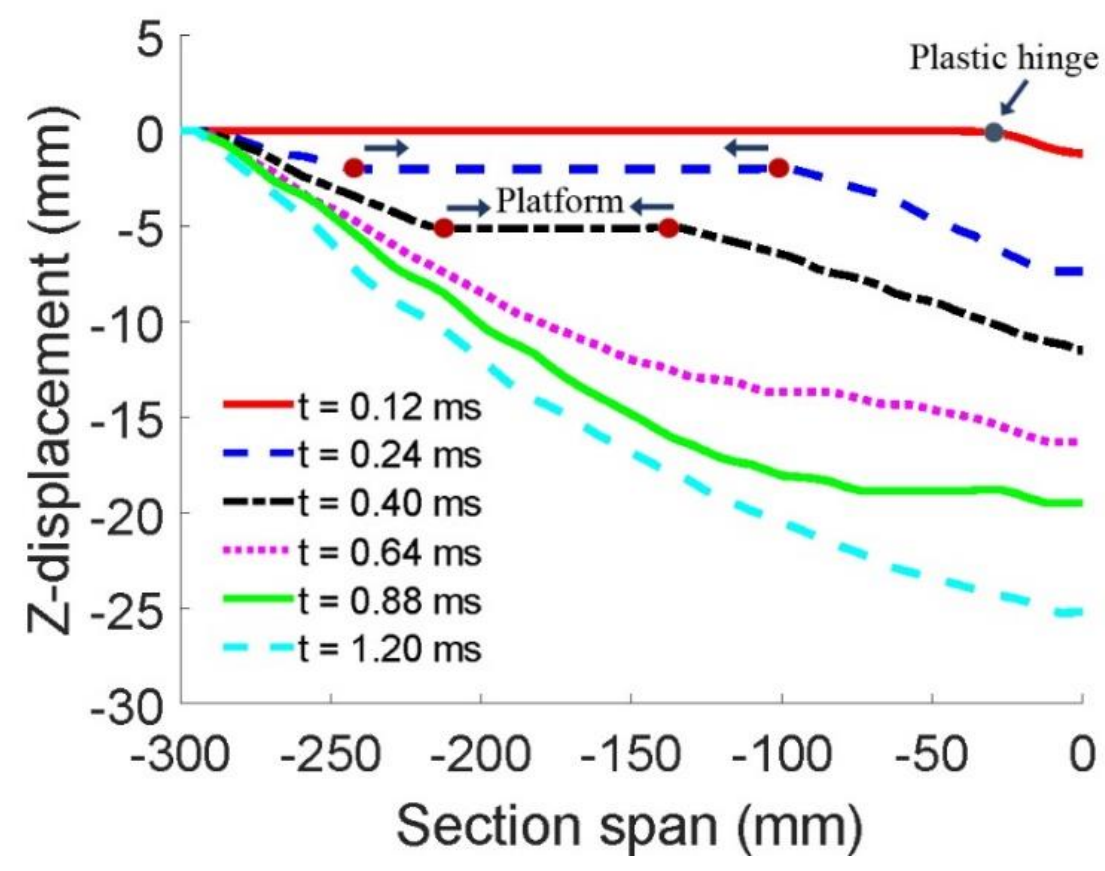

Fig. 14 Cross-section deformation profiles of UB model at different typical time.

The dynamic response process of plate under confined blast load was showed in Fig. 13, and the cross-section deformation profiles were depicted in Fig. 15. As shown in Fig.13, when subjected to unconfined blast load, slightly deformation occurred in the central area of the plate firstly. Dynamic response of the whole plate was observed in a very short time after the central area deformation (as shown in the $t=0.48 \mathrm{~ms}$ of Fig. 13). And then, larger plastic deformations were observed close to the boundaries then in the central area as shown in the $t=1.12 \mathrm{~ms}$ of Fig. 13 and Fig. 16, which was also observed in the experimental result as shown in Fig. 5). This phenomenon was caused by the converged, superimposed and intensified blast load in the corner of the box $[22,34]$. As time went by, the plastic deformation along the boundaries developed towards to the center (as shown in the Fig.13 from $t=0.88 \mathrm{~ms}$ to $t=1.44 \mathrm{~ms}$ ). At about $t=2.24 \mathrm{~ms}$ the deflection reached maximum value of about $46 \mathrm{~mm}$. 


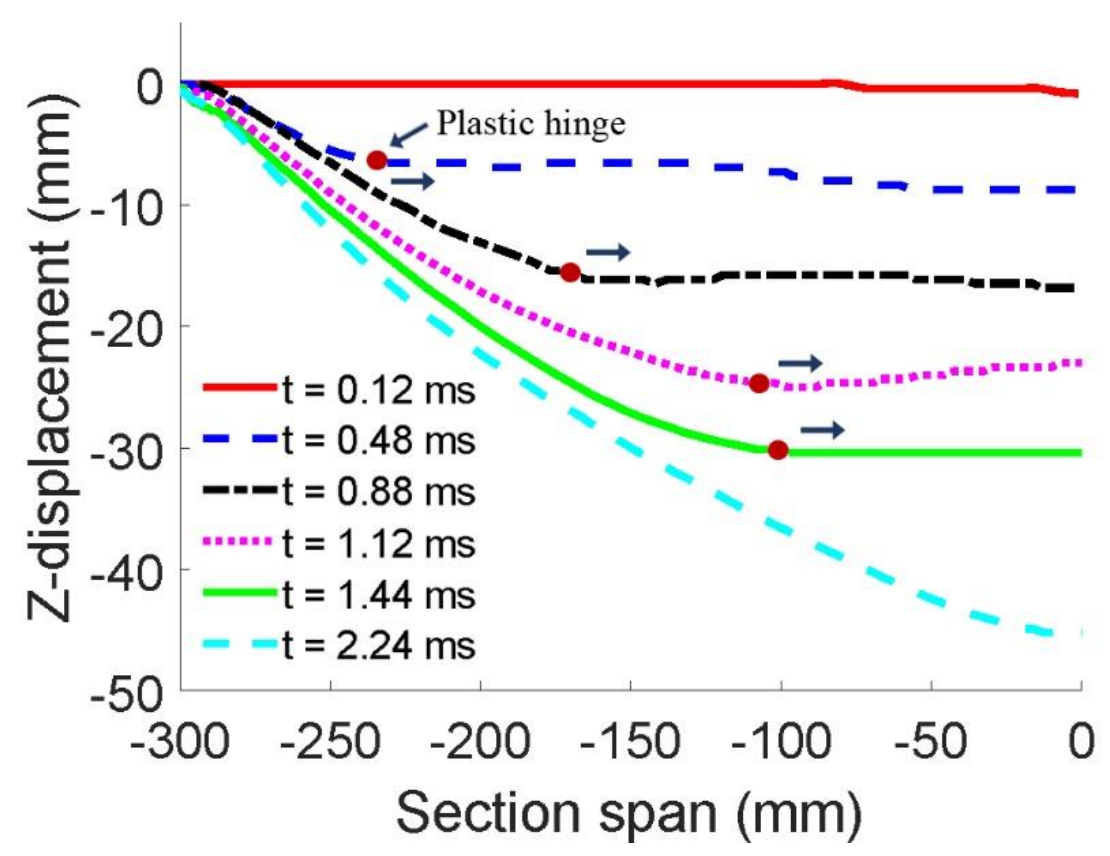

Fig. 15 Cross section deformation profiles of $\mathrm{CB}$ model at different time.
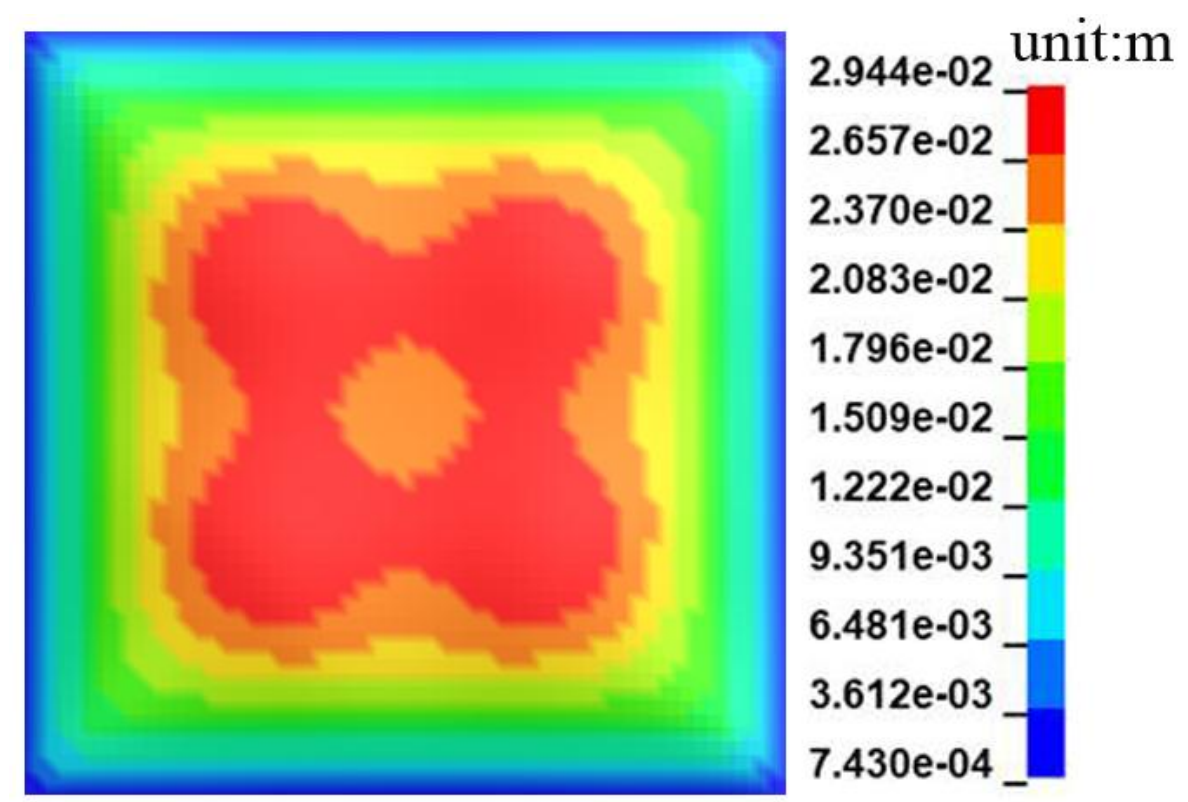

Fig. 16 Deformation feature of CB model at $t=1.12 \mathrm{~ms}$.

\section{Discussions}

From the studies of the dynamic response process of both FEM and DIC results, the dynamic response of the plate under UB load is very different from the CB load. The response mechanisms of plate under UB load and CB load are shown in Fig. 17. The dynamic response of plate under UB load can be divided into three phases as shown in Fig. 17(a). In phase I, the central region deforms, a plastic hinge circle forms around 
the deformed region, and the deformed region increases with the plastic hinge circle propagates radially. In phase II, new plastic hinge line occurs around the boundaries and propagates in the opposite direction to the central plastic hinge, and a platform remain undeformed between the boundary and the center. In phase III, the whole plate deformed, and deformation in the central area increased to the maximum value. The dynamic response of plate under CB load can be also divided into three phases as shown in Fig. 17(b). In phase I, the plastic hinge line happens in the boundaries, and propagates towards the plate center. A larger deformation in peripheral region than central area occurs in phase II. Same as the UB, maximum deformation happens in the central area at last, that's phase III.

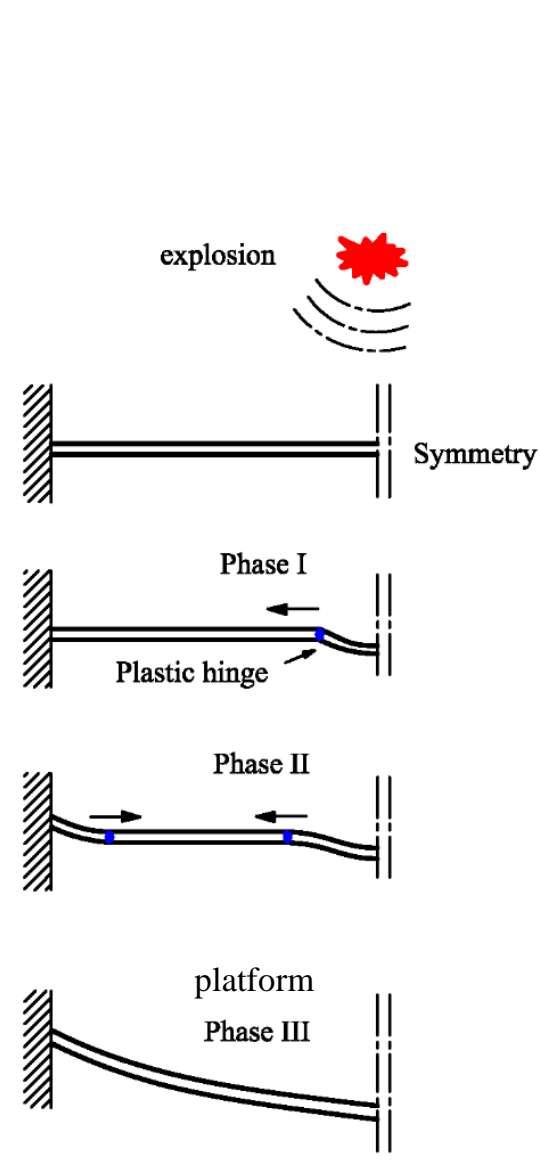

(a)
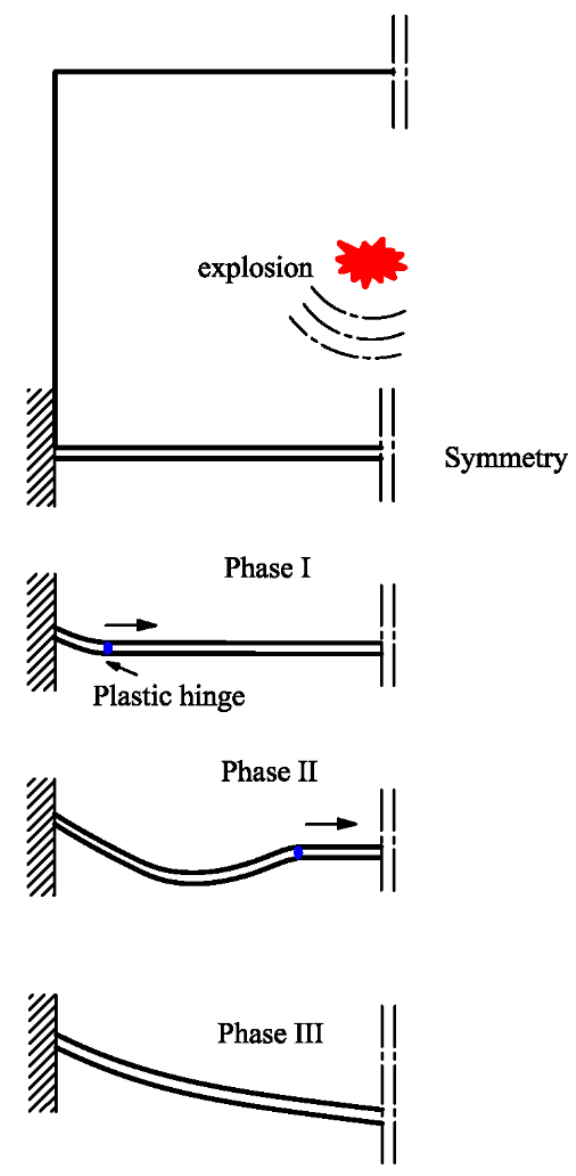

(b)

Fig. 17 Response mechanisms comparison between (a) UB and (b) CB.

\section{Conclusions}

According to the analyses mentioned above, some major conclusions can be drawn as below: 
a) The deformation features and dynamic response of steel plate under confined blast load were analyzed through both experimental and numerical results. The results show that outward bulging in side plate center and in-plane buckling in the middle position of boundary plates were both observes in numerical and experimental results. The DIC measured curve and the numerical calculated curves are similar in both the shape and the peak value, and the period of oscillation of numerical result fits well with the DIC result.

b) The dynamic response of steel plate under UB load and CB load were compared, the results showed that the dynamic response of plate can be divided into three phases under both UB and CB loads, while the dynamic response in phases I and II are different.

c) Differing from starting at the center and propagates to the boundary in case of UB condition, in phase I, plastic hinge in CB condition occurs close to the boundary and propagates in opposite direction. In phase II, two plastic hinge lines propagates towards each other, and a platform exists between the boundary and the center remain undeformed in $\mathrm{UB}$ condition, while in $\mathrm{CB}$ condition, a unique phenomenon of larger deformation in peripheral region than central area produces. All the achievements can provide guidelines for crashworthiness design of the kinds of defensive structures.

\section{Acknowledgement}

This research was funded by the Natural Science Foundation of China (51875581 and 11902369). The authors would like to express their thanks.

\section{References}

[1] Zhong D, Gong X, Han F, Li L. Monitoring the Dynamic Response of a Buried Polyethylene Pipe to a Blast Wave: An Experimental Study. Applied sciences 2019; 9(8), 1663.

[2] Curry RJ and Langdon GS. Transient response of steel plates subjected to close proximity explosive detonations in air. Int J Impact Eng 2017; 102: 102-116.

[3] Aune V, Fagerholt E, Hauge KO, Langseth M and Børvik T. Experimental study on the response of thin aluminium and steel plates subjected to airblast loading. Int J Impact Eng 2016; 90: 106-121.

[4] Nurick GN, Olson MD, Fagnan JR, et al. Deformation and tearing of blast-loaded stiffened square plates. Int J Impact Eng 1995; 16:273-291. 
[5] Nurick GN, Shave GC. The deformation and tearing of thin square plates subjected to impulsive loads-An experimental study. Int J Impact Eng 1996; 18(1):99-116.

[6] Bonorchis D, Nurick GN. The effect of welded boundaries on the response of rectangular hot-rolled mild steel plates subjected to localised blast loading. Int $J$ Impact Eng 2007; 34(11): 1729-1738.

[7] Bonorchis D, Nurick GN. The analysis and simulation of welded stiffener plates subjected to localised blast loading. Int J Impact Eng 2010; 37: 260-273.

[8] Chung Kim Yuen S, Butler A, Bornstein H, Cholet A. The influence of orientation of blast loading on quadrangular plates. Thin-Walled Struct 2018; 131: 827-837.

[9] Chung Kim Yuen S, Nurick GN. Experimental and numerical studies on the response of quadrangular stiffened plates. Part I: subjected to uniform blast load. Int J Impact Eng 2005; 31(1): 55-83.

[10] Jacob N, Nurick GN, Langdon GS. The effect of stand-off distance on the failure of fully clamped circular mild steel plates subjected to blast loads. Eng Struct 2007; 29(10):2723-2736.

[11] Mehreganian N, Louca LA, Langdon GS, Curry RJ, Abdul-Karim N. The response of mild steel and armour steel plates to localised air-blast loading-comparison of numerical modelling techniques. Int J Impact Eng 2018; 115:81-93.

[12] Yuan Y, Tan PJ. Deformation and failure of rectangular plates subjected to impulsive loadings. Int J Impact Eng 2013; 59:46-59.

[13] Mohammadzadeh B, Noh HC. An analytical and numerical investigation on the dynamic responses of steel plates considering the blast loads. Int J steel struct 2019; 19(2):603-17.

[14] Schiffer A, Tagarielli VL. The dynamic response of composite plates to underwater blast: Theoretical and numerical modelling. Int J Impact Eng 2014; 70:1-13.

[15]Latourte F, Gregoire D, Zenkert D, Wei X, Espinosa HD. Failure mechanisms in composite panels subjected to underwater impulsive loads. J Mech Phys Solids 2011; 59:1623-1646.

[16] Wang Z. Recent advances in novel metallic honeycomb structure. Composites Part $B$, 2019, 166: 731-741.

[17]Liu J, Wang Z, Hui D. Blast resistance and parametric study of sandwich structure consisting of honeycomb core filled with circular metallic tubes. Composites Part $B, 2018,145:$ 261-269. 
[18]Liu J, Chen W S, Hao H, Wang Z. Numerical study of low-speed impact response of sandwich panel with tube filled honeycomb core. Compos Struct 2019; 220:736748.

[19] Wang Z, Tian H, Lu Z, Zhou W. High-speed axial impact of aluminum honeycomb-Experiments and simulations. Composites Part B. 2014; 56:1-8.

[20] Wang Z, Li Z, Shi C, Zhou W. Theoretical and numerical analysis of the folding mechanism of vertex-based hierarchical honeycomb structure. Mechanics of Mech Adv Mater Struc. 2019:1-1.

[21]Dragos J, Wu CQ, Oehlers DJ. Simplification of fully confined blasts for structural response analysis. Eng Struct 2013; 56:312-326.

[22] Yao SJ, Zhang D, Lu FY, Li XC. Experimental and numerical studies on the failure modes of steel cabin structure subjected to internal blast loading. Int J Impact Eng 2017; 110:279-287.

[23] Yao SJ, Zhang D, Lu FY. Dimensionless number for dynamic response analysis of box-shaped structures under internal blast loading. Int J Impact Eng 2016; 98:1318.

[24]Fay SD, Rigby SE, Tyas A, Clarke SD, Reay JJ, et al. Displacement timer pins: An experimental method for measuring the dynamic deformation of explosively loaded plates. Int J Impact Eng 2015; 86: 124-130.

[25] Yao SJ, Zhang D, Lu FY, Wang W, Chen XG. Damage features and dynamic response of RC beams under blast. Eng Fail Anal 2016; 62: 103-111.

[26] Yao SJ, Zhang D, Chen XG, Lu FY, Wang W. Experimental and numerical study on the dynamic response of RC slabs under blast loading. Eng Fail Anal 2016; 66: 120-129.

[27] Helm JD, McNeill SR, Sutton MA. Improved 3D image correlation for surface displacement measurement. Opt Eng 1996;35(7):1911-20.

[28] Sutton MA, Orteu J, Schreier HW. Image correlation for shape, motion and deformation measurements. USA: Springer; 2009.

[29] Tiwari V, Sutton MA, McNeill SR, Xu S, Deng X, et al. Application of 3D image correlation for full-field transient plate deformation measurements during blast loading. Int J Impact Eng 2009; 36:862-874. 
[30]Rigby SE, Tyas A, Curry RJ, Langdon GS. Experimental Measurement of Specific Impulse Distribution and Transient Deformation of Plates Subjected to Near-Field Explosive Blasts. Experimental Mechanics 2019; 59(2):163-178.

[31] Spranghers K, Vasilakos I, Lecompte D, Sol H, Vantomme J. Numerical simulation and experimental validation of the dynamic response of aluminum plates under free air explosions. Int J Impact Eng 2013; 54:83-95.

[32] Kumar P, LeBlanc J, Stargel DS, Shukla A. Effect of plate curvature on blast response of aluminum panels. Int J Impact Eng 2012; 46:74-85.

[33] Wu J, Li L, Du XL, Liu XM. Numerical Study on the Asphalt Concrete Structure for Blast and Impact Load Using the Karagozian and Case Concrete Model. Applied Sciences. 2017;7(2):202.

[34] Yao SJ, Zhang D, Lu ZJ, Lin YL, Lu FY. Experimental and numerical investigation on the dynamic response of steel chamber under internal blast. Eng Struct 2018; 168:877-888.

[35]LS-DYNA keyword user's manual. Livermore Software Technology Corporation, 2007.

[36] Johnson GR, Cook WH. A constitutive model and data for metals subjected to large strains, high strain rate and high temperatures. Proceeding of the 7th international symposium on ballistics, Hugue, Netherlands, 1983.

[37] Ma J, Fan F and Zhi X. Counter-intuitive collapse of single-layer reticulated domes subject to interior blast loading. Thin-Walled Struct 2015; 96: 130-138.

[38]Dong Q, Li QM and Zheng JY. Further study on the counter-intuitive response of single-degree-of-freedom structures. Int J Impact Eng 2011; 38: 305-308.

[39]Flores-Johnson EA and Li QM. A brief note on the counter-intuitive region of a square plate. Int J Impact Eng 2010; 38: 136-142. 